Historic, Archive Document

Do not assume content reflects current scientific knowledge, policies, or practices. 


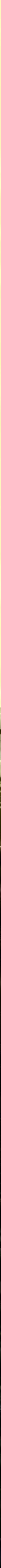
WeLLeR NURsERIES CO., INC.

HOLLAND, MICHIGAN
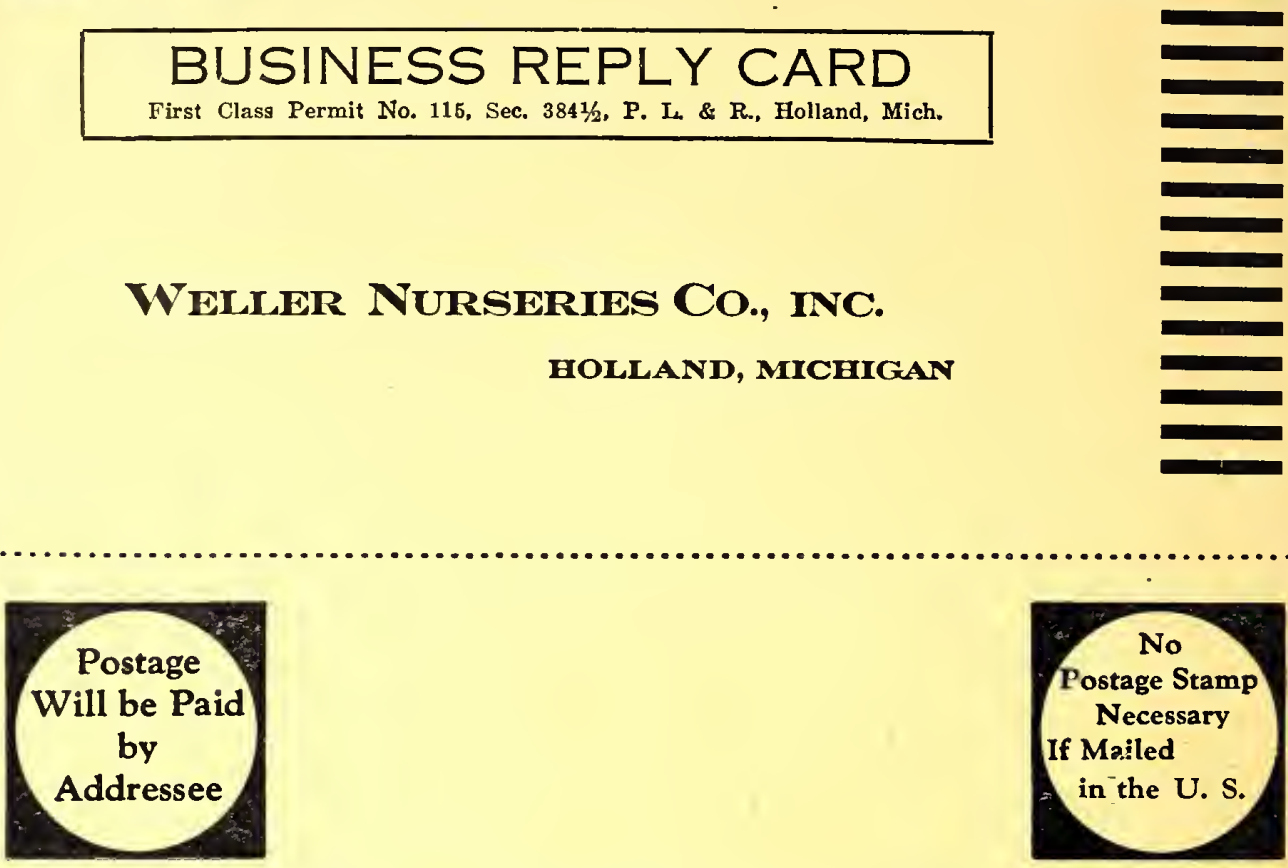

\section{BUSINESS REPLY CARD}

First Class Permit No. 115, Sec. 3841/2, P. L. \& R., Holland, Mich.

Weller Nurseries Co., inc.

HOLLAND, MICHIGAN

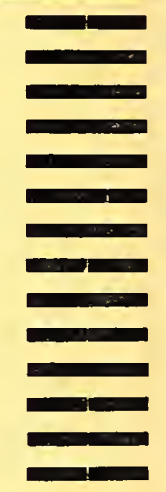

\section{NEW AND IMPORTED VARIETIES: NOVELTIES AND RARE SPECIES} WHICH YOU SHOULD GROW

Varieties marked with (*) are good for cut-flowers.

*AQUILEGIA HELENEA. Beautiful, very large flower. Outer petals deep blue, center pure white. 3 ft. May-June ....................................................................................

ARTEMISIA SILVER KING. Striking silvery white foliage, making a good bush. Good

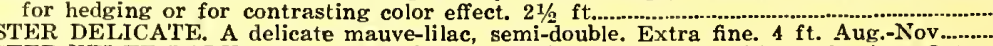
*ASTER DELICATE. A delicate mauve-lilac, semi-double. Extra fine. $4 \mathrm{ft}$. Aug.-Nov...... CAMPANULA LATILOBA GRANDIS. Broad clustered bell-shaped fowers of clear blue.

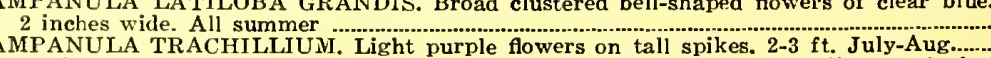

CAMPANULA TRACHILLIUM. Light purple flowers on tall spikes. 2-3 $\mathrm{ft}$. July-Aug......
CLEMATIS TANGUTICA (New) Flowers solitary, bright yellow, nodding, 2 inches accoss. A fine climber growing $10 \mathrm{ft}$. high. Flowering in June-July. Later in the summer large heads of feathery fruits. Very handsome..............................................

GAILLARDIA PORTOLA HYBRIDS. Brilliant scarlet, with copper cones. 2 ft. All summer.
UNKIA ROBUSTA ELEGANS FOLIO VARIEGATA. A very robust grower with beautifully strong variegated foliage. Much better than Undulata Medio Variegata..............

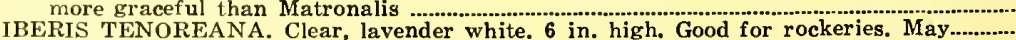
*LYTHRUM SALICARIA. Huge pyramids of rose-purple fowers. 2-3 ft. All summer.....

* LYTHRUM SALICARIA ATROPURPUREA. Dark rose-purple. 2-3 ft. All summer........... MERTENSIA PANICULATA. An old and scarce variety, but very beautiful. Flowers deep blue, in loosely panicled racemes.....................................................................

PENSTEMON PUBESCENS. Stems 24 in. bigh, covered with violet flowers shading to

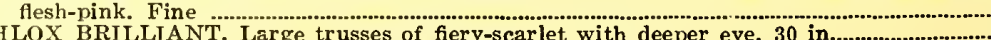
PHLOX MRS. ETHEL PRITCH ARD. Iare trusses of even mauve. The best blue Phlox.

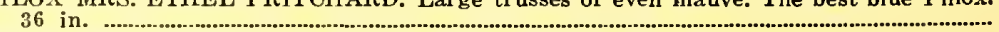
H 6 X MS. RUTGERS. Beautiful, light lilac-pink, dark center. PHLOX MRS. SCHOLTEN. Dark salmon-orange. Large and very fine........................................ 2.00 PHLOX WM KESSELRING Large, violet with white eye

POTENTILLA CHRYSOCRASPEDA. Golden yellow growing 6 in. high. Fine for rocker-

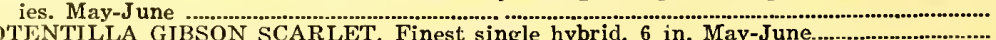

POTWNTILLA GIBSON SCARLET. Finest single hybrid. 6 in. May-June............... varieties
SHASTA DAISY ALASKA LACINIATA. Large flower with pure white plume-shaped

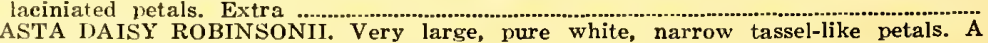

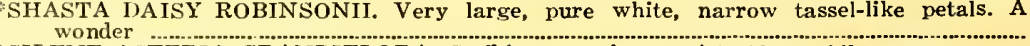

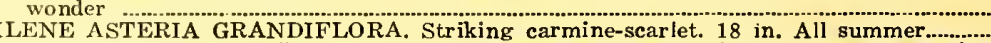

VERONICA LATIFOLIA ROYAL BLUE. A beautiful dwarf variety, profusely flowering. Deep blue 6 inch. June-July

Per 10 Per 100 $1.75 \quad 15.00$ $2.50 \quad 20.00$ $2.75 \quad 25.00$ $2.50-20.00$ 50.00 15.00 20.00 15.00 15.00 15.00 15.00 50.00 15.00 20.00 25.00 


\section{ROCK AND ALPINE PLANTS}

The demand for Rock and Alpine Plants is without question a prominent one and here to stay. You where people can see them and they will buy.

STRONG FIELD-GROWN STOCK

Per 10 Per 100

AJUGA REPTANS. Deep purplish flowers. $3-4$ inch. June ALYSSUM ARGENTEUM. Silvery leaves, yellow

ALYSSUM AROSTRATUM. Pale Yellow, June

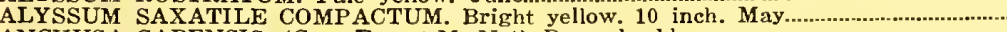

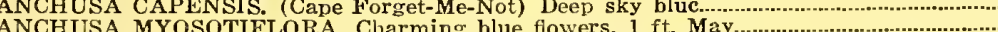

ANCHUSA MYOSOTIFLORA. Charming blue flowers. 1 ft. May

AQUILEGIA (Columbine) ALPINA. Dwarf, mixed colors. Jun

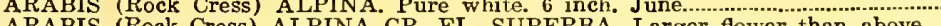

ARABIS (Rock Cress) ALPINA GR. FL. .SUPLRB. Larger flower than above..............................

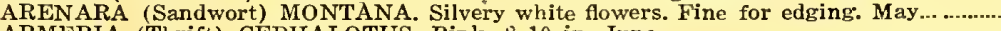

ARMERIA (Thrift) CEPHALOTUS. Pink. 8-10 in. June

ARMERIA (Thrift) FORMOSA. Rose. 9 inch. June....................

ARMERIA (Thrift) MARITIMA Pale Dink. 3-6 inch. J Je- June.

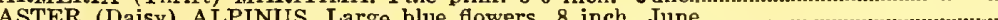

AUBRIETIA (Rock Cress) HENDERSONII Violet Creeping

BELLIS (English Daisy) PERENNE Pink, red and white

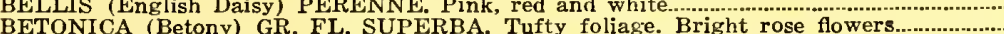

CALAMINTHA (Calamint) ALPINA. Rich purple, 6 inch June rose flow

CAMPANULA (Harebell) CARPATHICA. Blue. 8 inch. June

CAMPANULA (Harebell) CARPATHICA ALBA. White variety

CAMPANULA (Blue Bells of Scotland) ROTUNDIFOLIA. Clear blue. 10 inch. June-Aug.

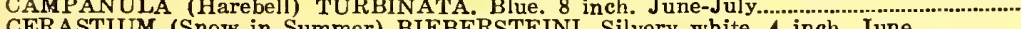
CERASTIUM (Snow in Summer) BIEBERSTEINI. Silvery white. 4 inch. June...................

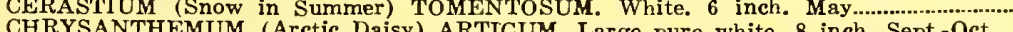
CHRYSANTHEMUM (Arctic Daisy) ARTICUM. Large pure white. 8 inch. Sept.-Oct.....

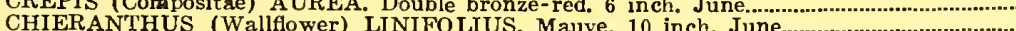

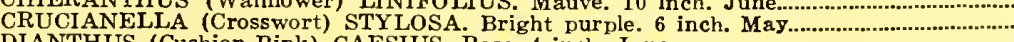

DIANTHUS (Maiden Pink) DELTOIDES. Crimson. 6 inch. July.

DIANTHUS (Maiden Fink) DELTOIDES BRILLIANT Glowing crimson..................... 6 inch. July EUPHORBIA POLYCHROMA. Pretty green foliage. Yellow flowers. $1 \mathrm{ft}$. May-June......... FESTUCA (Blue Fescue Grass) GLAUCA. Fine for edging. 12 inch

*HELIANTHEMUM (Sunrose) MUTABIIIS. A mixture of pink and orange. 8 inch. JulySept. .

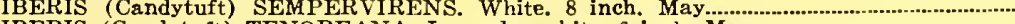
IBERIS (Candytuft) TENOREANA. Lavender white. 6 inch. May.

IRIS PUMILA CYANEA. Dwarf Iris. Deep blue. Ma

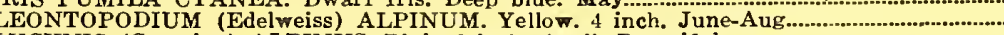

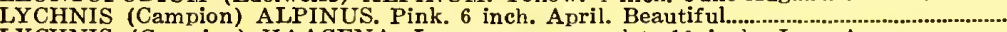
LYCHNIS (Campion) HAAGENA. Large orange-scarlet. 10 inch. June-Aug.................... MYOSOTIS (Forget-Me-Not) PALUSTRIS SEM PERFLORENS.

PAPAVER (Iceland Poppy) NUDICAULE. Striking colors of orange, yellow and white.

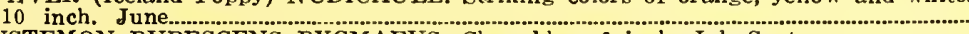
PENSTEMON PUBESCENS PYGMAEUS. Clear blue 6 inch July-Sept..........

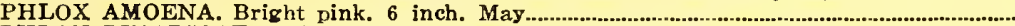

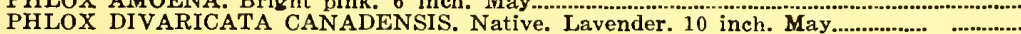

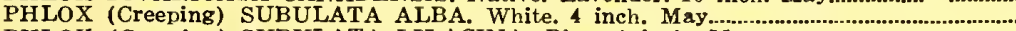

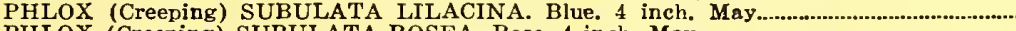

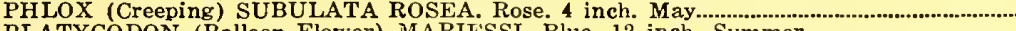

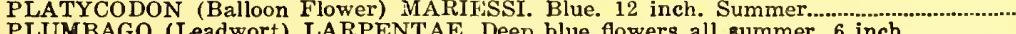

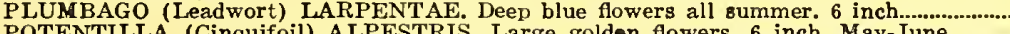
POTENTILLA (Cinquifoil) ALPESTRIS. Large golden flowers. 6 inch. May-June............ POTENTILLA (Cinquifoil) ATROSANGUINEA. Beautiful scarlet. 6 inch May-June...... POTENTILLA (Cinquifoil) CHRYSOCRASPIDA. Golden yellow. 6 inch. May-June............. POTENTILLA (Cinquifoil) FORMOSA. Red. 8 inch. All summer....................................... POTENTILLA (Cinquifoil) GIBSON SCARLET. Finest single hyrid. 6 inch. May-June...

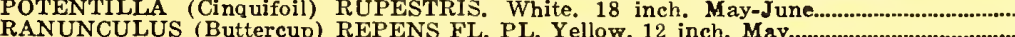

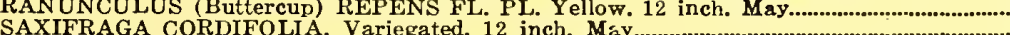
SEDUM (Golden Moss) ACRE. Yellow, 4 inch, June SEDUM (Spreading Moss) ALBUM. White. 4 inch. Junc..................................................................... SEDUM (Blue Moss) GLAUCUM. Blue foliage. 4 inch

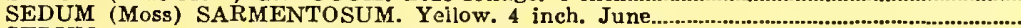

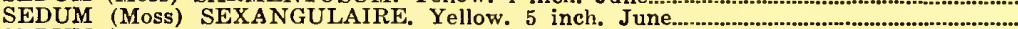

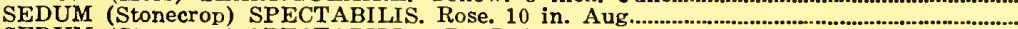

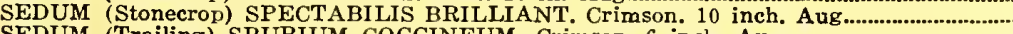

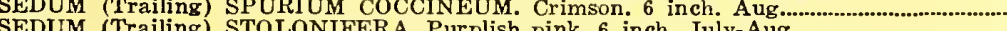

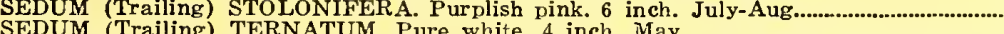
SEMPERVIVUM (Hen and Chickens) SOBOLIFERUM. Light green foliage, flowers SILENE (Autumn Catchtly) SHAFTA. Bright pink. 6 inch. July-Oct. STOKESIA (Stokes Aster) CYANEA. Blue. 12 inch. June-Oct................................................... SPERGULA PILIFERA. White 2 inch. June - .

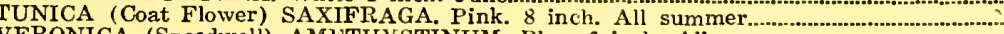

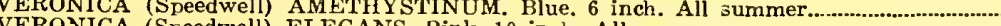
VRONICA (Speedwell) INCANA. Pall blue 9 in. June-July VERONICA (Seedwell) INCANA. Pale blue. inch. June-July

VERTICA (SpD

VIOLA (Tufted Pansy) ADMIRATION Light blue

VIOLA (Tufted Pansy) BLACK PRINCE Velvety purple.

VIOLA (Tufted Pansy) JERSEY GEM. Violet. Fxtra fine.

VIOLA (Tufted Pansy) LUTEA SPLENDENS, Yellow

VIOLA (Tuf ted Pansy) MAUVE QUEEN. Mauve...................................

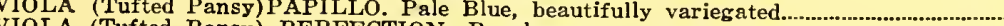

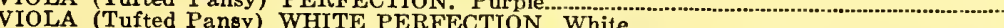

VINCA (Periwinkle or Myrtle) Minor. Blue flowers............................................................. 1.25

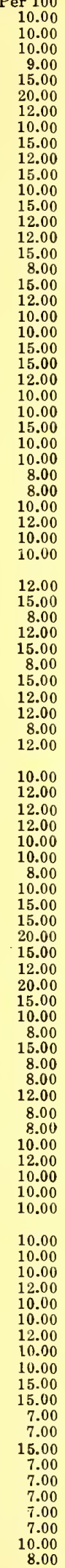

\section{DAISY AND MARGUERITE FLOWERING PERENNIALS}

Such as: Hardy Asters, Boltonia, Coreopsis, Gaillardia, Helenium, Sunflowers, Cone Flower, Shasta an, etc.

Varieties marked with (*) are good for cutting.

ASTER (Michaelmas Daisy)

ASTER ABENDROTHE. Beautiful deep rose. $5 \mathrm{ft}$.

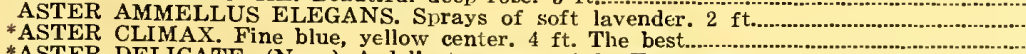

*ASTER ELTA. Clear lavender.

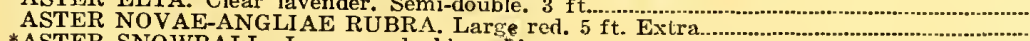

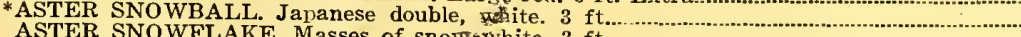

ASTER SNOW QUEEN. Pure white, 2-3 $\mathrm{ft}$

ASTER ST. EGWIN. Large, clear pink. 2 ft.... 


\section{DAISY AND MARGUERITE FLOWERING PLANTS}

*ASTER White LADY. (New.) The best and largest white.

Per 10 Per 100

ASTER WHITE QUEEN. White. 34 ft.TIES- $\$ 10.00$ per $100 ; \$ 80.00$ per 1000 .

*ANTHEMIS (Margucrite) KELWAYI. Clear yellow. 2 ft. June-July..................................... 1.00

BOLTONIA (Starwort) ASTEROIDES. White. 5-6 ft. Aug. Sept...- 1.00

BOLTONIA (Starwort) LATISQUAMA. Bright pink. $4 \mathrm{ft}$. Aug .................................... * COREOPSIS (Tickseed) GRANDIFLORA. Yellow. $2 \mathrm{ft}$. Summer

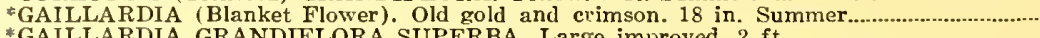
*GAILLARDIA GRANDIFLORA SUPERBA. Large improved. 2 ft.......................................... * GAILlLARDIA LE MASTADONTE. Large, pure yellow. $2 \mathrm{ft}$.......... *GAILlLARDIA PORTOLA HYBRIDS. Brilliant scarlet.

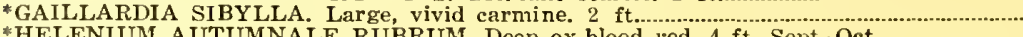
*HEI *HELENIUM HOOPESI. Earliest flowering. Pure orange. 2-3 ft. May-June * HETIANTHUS (Sunflor) MISS MELIT. Pure orange. 2-3 At. May-J * HELIOPSIS (Orange Sunfower) PITCHERIANA. Golden yellow. $3 \mathrm{ft}$. All summer

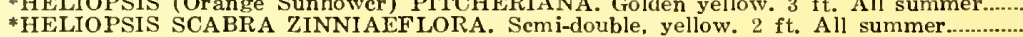

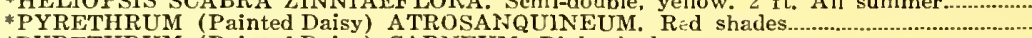
*PYRETHRUM (Painted Daisy) CARNEUM. Pink shades

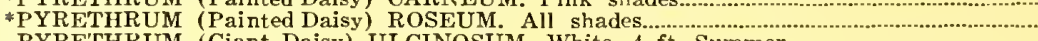
PYRE'THRUM (Giant Daisy) ULGINOSUM. White. $4 \mathrm{ft}$. Summer *SHASTA DAISY ALASKA. Large white

* SHASTA DAISY ALASKA CONQUEROR. Largest in existence..

* SHASTA DAISY ALASKA KING EDW. VII. Hardiest of all............... *SHASTA DAISY ALASKA LACINIATA. Large flower, pure white, plume shaped, laciniated petals. Extra ................. white, earliest of all.
*SHASTA DAISY ELDER. Large,

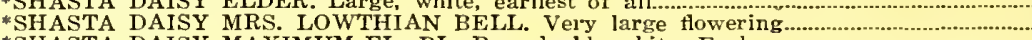
*SHASTA DAISY MAXIMUM ROBINSONII. Very large flower, narrow tassel-like petals. A wonder .............................................................................................................

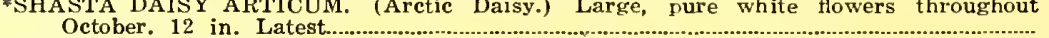
* RUDBECKIA (Black-eyed Susan) NEWMANNI. Yellow with brown center. $2 \mathrm{ft}$. All

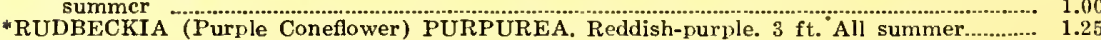

IRIS KAEMPFERI (Japanese Iris)

ASTARTE. Double, dark violet

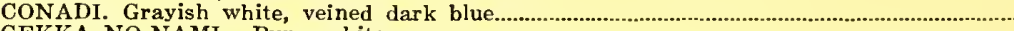

GEKKA-NO-NAMI. Pure white

GOLD BOUND. Double, white and yellow

HELEN VON SIEBOLD. Bright crimson, shading into purple

HERCULE. Clear, lilac-blue ......................................

KUMO-FUNGI. Purple, navy blue, yellow

作

A.

MULPI

PYRAMID Corulean blue purple

SHIGA-NO-URANAMI. Purplish crimson and lavender

SUFO-NO-KOI. Grey-lilac, flaked with light lavender.

UCHIU. Coerulean blue, golden center

FINE MIXTURE- $\$ 75$ per 1000 .

ABOVE NAMED VARIETIES- $\$ 125.00$ per 1000 .

\section{WATER IRIS}

IRIS PSEUDACORUS. (Waterflag) Yellow, shaded orange. $3 \mathrm{ft}$. May-June........................ 1.50

IRIS GERMANICA (Liberty Iris)

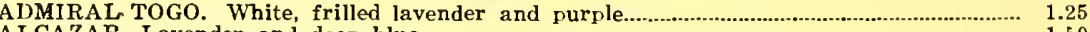
ALCAZAR. Lavender and deep blue. AMAS. Light blue and velvety-violet........e..... AMERPOHL Lavender and deep blue

ANNA LESLlE. White, tinted rose. Dahlia carmine

AUREA. Deep golden yellow.

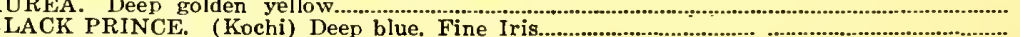
CANDALA BRA. Silvery mauve ..................

CAPRISE. Reddish purple. The best red.....

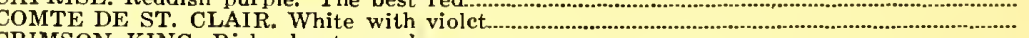

DAWN. Sulphur yellow, veined prone.

DOROTHY

EDITH COOK Yellow and purple.

ELDORADO. Bronze yellow and violet.

FAIRY. Lovely white with blue shadings

FLAVESCENS. Yellow

GAJUS. Light yellow and crimson

GERTRUDE. Clear violet blue

GYPSY QUEEN. Old gold and crimson

HER MAJESTY. Rose pink, darker veined.

ISONORABILIS. Golden yellow and brown...

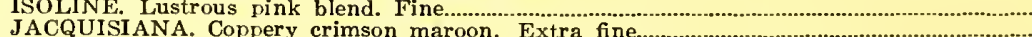

JAN crimson maroon. Extra fine.

JUANITA ARC. White, frilled with lilac-blue...

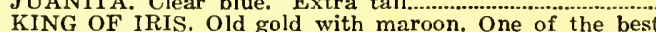

ANY

LAVENDER. A beautiful lavender, large flower

L'AVENIR. A beautiful shade of satin-blue.

E KHEDIVE. Soft lavender and white

LOHENGRIN. Silvery mauve

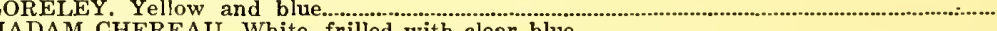

MADAM CHEREAU. White, frilled with clear blue

MADAM. Ruffled white, delicately peneiled blue

MARY GARDEN. Attractive pale yellow, speckled lavender

.

(D.

PALLIDA SPECIOSA. Deep purple

PARC DE NEUILLY. Deep purple..

PERFECTION LiLY. Deep violet-bor

REMBRANDT Soft lilac blue

RHEIN NIXE. Pure white with deep violet blue Beautiful.

QUEEN OF MAY, Lavender pink

SHAKESPEARE. Bronze-yellow and maroon

SHERWIN WRIGHT. The best golden yellow

STORM CLOUD. Slate blue with bronze...

WHTE KNIGHT. Fine snow-white Iris. Sweet scented

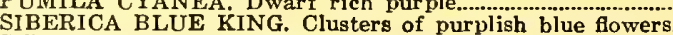

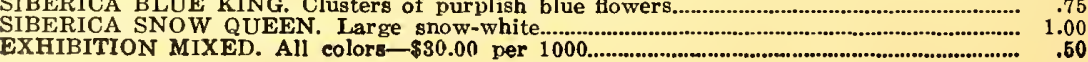




\section{GENERAL LIST OF PERENNIALS}

\section{For the Hardy Border and Cut-Flower Purposes}

In selecting a general line of Perennials for all purposes, please consult all other groups listed. The necessity of florists having "a pateh of Perennials for cut-flowers" is beyond question. It furnishes a continuous supply of flowers when other fowers are scarce within your immediate reach. No ordering, waiting nor express charges and bils to pay. You will be surprised what it will do for you.
Do not forget varieties under Novelties and Daisies when ordering. STRONG, FIELD-GROWN STOCK

All varities suitable for cut-flower purposes are marked with a $(*)$.

Per 10 Per 100

*ACHILLEA BOULE DE NEIGE. Double white. $2 \mathrm{ft}$. June-Sept...

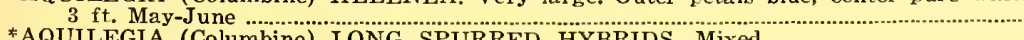

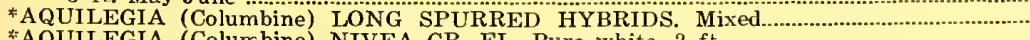
AQUILEGIA (Columbine) NIVEA GR. FL. Pure white. $2 \mathrm{ft}$.

*AQUILEGIA (Columbine) ROSE QUEEN. Pink shades, long spurs. $21 / 2 \mathrm{ft}$. May-June. *ARMERIA (Thrift) FORMOSA. Flesh to deep pink. 9 in. June

"ARTEMISIA LACTIFLORA. White feathery flowers. $4 \mathrm{ft}$. Aug..

"ARTEMISIA SILVER KING. Silvery foliage. $21 / 2 \mathrm{ft}$. Good......

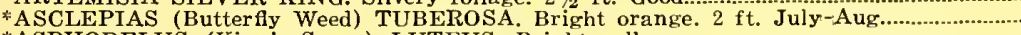
*ASPHODELUS (King's Spear) LUTEUS. Bright yellow

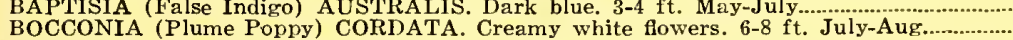

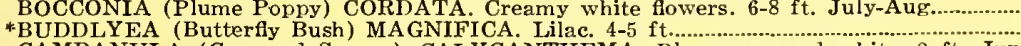
CAMPANULA (Cup and Saucer) CALYCANTHEMA. Blue, rose and white. $2 \mathrm{ft}$. Jun *CAMPANULA LATILOBA GRANDIS. Broad clustered bell-shaped flowers. Blue. 2 in CAMPANULA (Canterbury Bell) MEDIUM. Dark blue, light blue, rose and white. 2 ft.

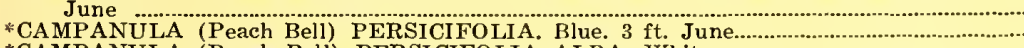
*CAMPANULA (Peach Bell) PERSICIFOLIA ALBA. White CAMPANULA (Chimney Bell) PYRAMIDALIS. Blue. 4-5 ft. Aug. "CAMPANULA (Coventry Bell) TRACHELIUM. Light purple. "CARNATION GIANT FLOWERING. Mixed ce

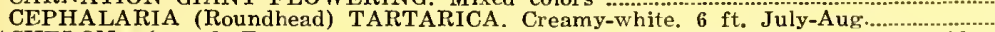
*CHELONE (Beards Tongue) BARBATUS HYBRIDS. A fine mixed strain of Hybrids. *CHELONE (Beards Tongue) BARBATUS TORREYI. Scarlet. 3-4 ft. July-Aug. * CATANANCHE (Cupid's Dart) COERŨLA. Blue Alowered. 2 ft. June-Au *CENTAUREA (Knapweed) DEA LBATA. Striking rose-pink, $2 \mathrm{ft}$. June-July "CENTAUREA MACROCEPHALA. Golden yellow. $3 \mathrm{ft}$. July-Aug. C. Conta *CIMICIFUGA (Snakeroot) RACEMOSA Tall,

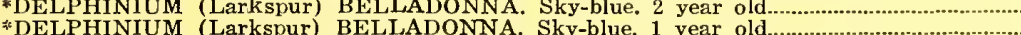
*DELPHINIUM (Larkspur) BELLAMOSA. Deep blue, 2 year old *DELPHINIUM (Larkspur BELLAMOSA. Deep blue, 1 year old.

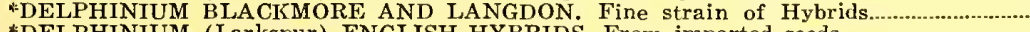
DELPHINIUM (Larkspur) ENGLISH HYBRIDS. From imported seeds.

2-year old

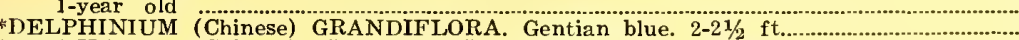

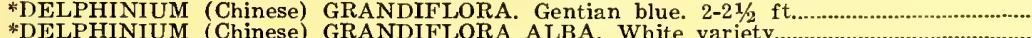
DIANTHUS BARBATUS (See Sweet William)

*DIANTHUS (Hardy Pink) PLUMARIUS. Single mixed. $1 \mathrm{ft}$. All summer.

:DIANTHUS (Hardy Pink) SEMPERF LORENS. Everblooming mixed.......................... DIELYTRA (Bleeding Hearts) SPECTABILIS. Old-fashioned favorite

DIGITALIS (Foxglove) AMBIGUA. Yellow, hardy .................................................................. DIGITALIS (Foxglove) GLOXINAEFLORA. Purple, rose and white......

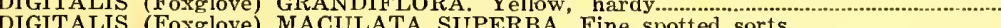

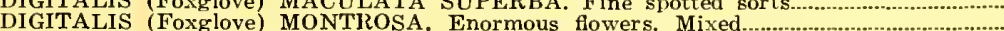
DICITALIS (Fxglove) MONTROS. Enormous flowers. Mixed.

*ECHINOPS (Globe Thistle) RITRO. Metallic blue flowers. 2-3 ft *ERYNGIUM (Sea Holly) AMETHYSTINUM. True blue Thistle

*EUPATORIUM (Hardy Ageratum) COELESTIUM. Lavender blue. 18 in. From August till frost *EUPHORBIA (Milk Wort) COROLATA. Like Gypsophilla. Pure white....................................

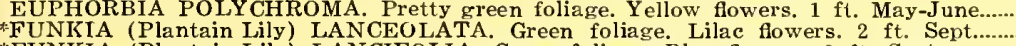

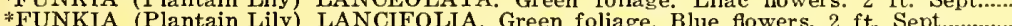
FUNKIA (Plantain Lily) MEDIO VARIEGATA. Variegated leaves

*FUNKIA ROBUSTA ELEGANS FOL. VARIEGATA. A very robust grower with beauti

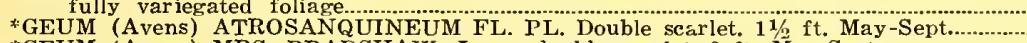

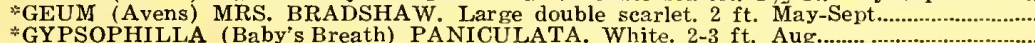

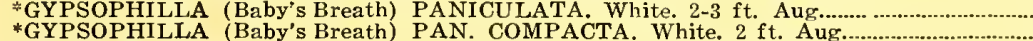
GRASS GRASS ELYMUS (Blue Lime Grass) GLAUCUS. Silvery blue leaves. Very ornamenta and pretty $\ldots \ldots$

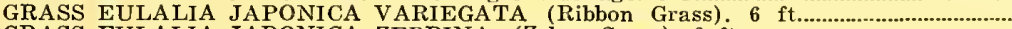

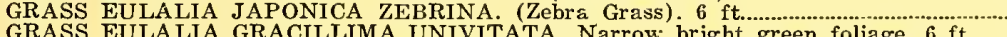
GRASS EULALIA GRACILLIMA UNIVITATA. Narrow bright green foliage. $6 \mathrm{ft}$...................... GRASS FESTUCA GLAUCA. Fine blue foliage. 12-15 in............................................................. GRASS STIPA SPLENDENS (Awned Grass). Very ornamental. 4-5 ft..................... *HEMEROCALIIS (Day Lily) KWANSO FL. PL. Large, double, rich golden bronze.

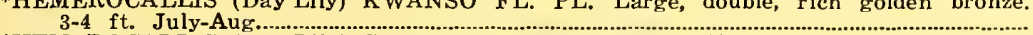

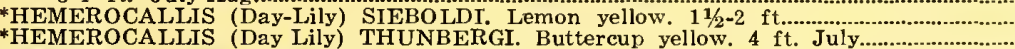
*HESPERIS (Dames Violet) LAMSONIFOLIA. (New.) A beautiful, improved variety.

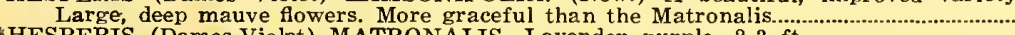

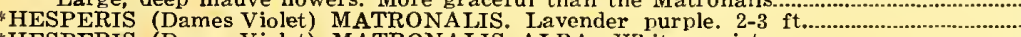

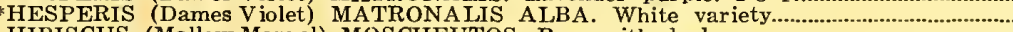
HIBISCUS (Mallow Marvel) MOSCHEUTOS. Rose with darker eye............................................. HIBISCUS NEW GIANT MALLOWS. Red, pink and white...........................................................
HOLLYHOCK DOUBLE VARIETIES. Apple blossom, maroon, red, rose, salmon, white

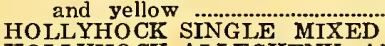

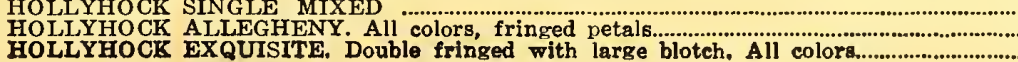

12.00

15.00

10.00

12.00

12.00

10.00

10.00

20.00
12.00

12.00

10.00

10.00

20.00

10.00

20.00

10.00 
LIATRIS (Blazing Star) SPICATA Purple spikes, $2-3 \mathrm{ft}$ July-Sent *LILIUM (Tiger Lily) TIGRINUM SIMPLEX. Single, brilliant orange. LINUM (Perennial Flax) PERENNE. Blue flowers. 11/2 $\mathrm{ft}$. May-June

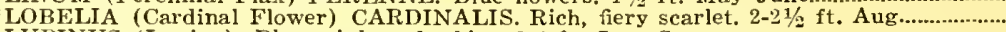

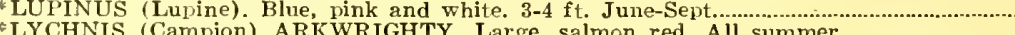

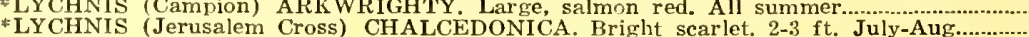
LYCHNIS (Jerusalem Cross) CHALCEDONICA. Bright scarlet. 2-3 ft. July-Aug..........................

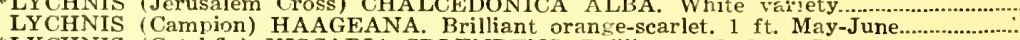

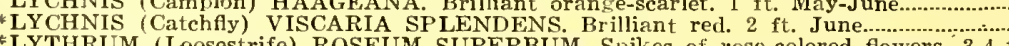
LYTHRUM (Loosestrife) ROSEUM SUPERBUM. Spikes of rose-colored flowers. 3-4

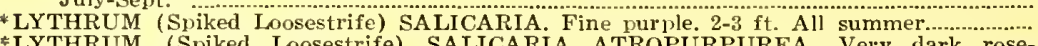
LYTHRUM (Spiked Loosestrife) SALICARIA ATROPURPUREA. Very dark rose

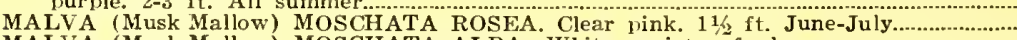
MALVA (Musk Mallow) MOSCHATA ALBA. White variety of above

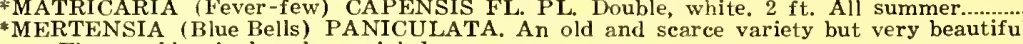

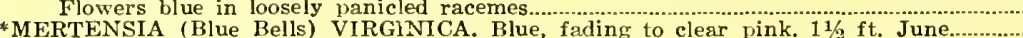

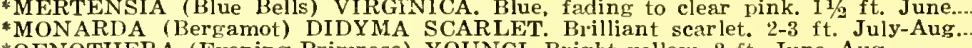

OENOTHERA (Evening Primrose) YOUNGI. Bright yellow. 2 ft. June-Aug..................... PAPAVER (Iceland Poppy) NUDICAULE. Mixed orange, yellow and white $12 \mathrm{in}$. Jun

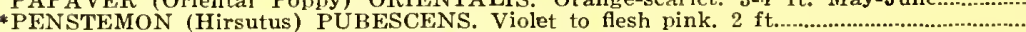

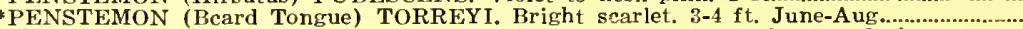

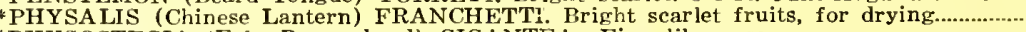

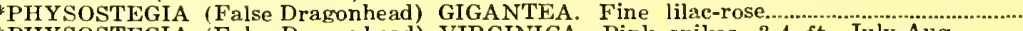

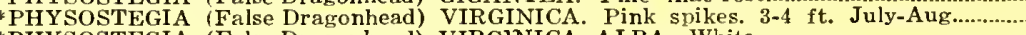
*PHYSOSTEGIA (False Dragonhead) VIRGINICA ALBA. White

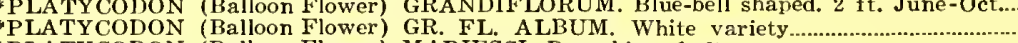

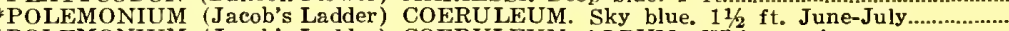

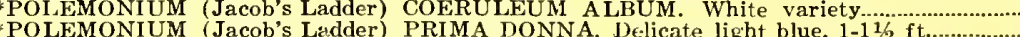
* POLEMONIUM (Golden Glow) LACINIATA FL. PL. Clear double yellow. 5-6 ft. July-Sept. RUDBECKIA (Golden Glow) LACINIATA FL. PL. Clear double yellow. 5-6 ft. July-Sept.

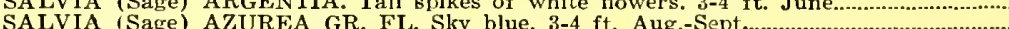
SAIVIA (Sage) PRATENSIS. Deep blue. Extra fine 2 ft June.

SAIVIA (Sage) GLOBOSA white floe. Ext in fun

"SALVIA TURKESTANICA. Very showy large spikes of white flower spikes, surrounded

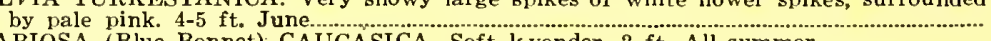
* SCABIOSA (Blue Bonnet) CAUCASICA. Soft lavender. $2 \mathrm{ft}$. All summer........................ * SIDALCEA (Greek Mallow) ROSE QUEEN. Bright rose flowers. Extra fine. 2-3 ft. June-July

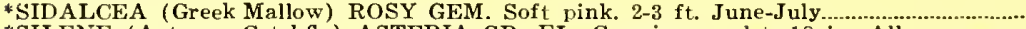
*SILENE (Autumn Catchfly) ASTERIA GR. FL. Carmine scarlet. 18 in. All summer.....

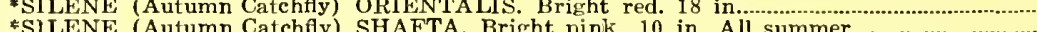

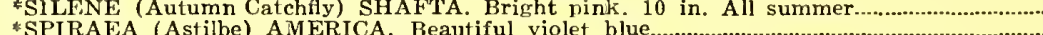

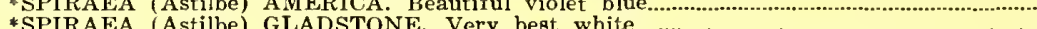
SPIRAEA (Astibe) GLORIA De. Very best

* SPIRAEA (Astibe) GLORA . Deep SOM .

*SPIRAEA (Astilbe) QUEEN ALEX ANDRA Cloar pink.

. * STATICE (Sea Lavender) INCANA HYBR ROSA. Pink

*STATICE (Sea Lavender) LATIFOLIA. Lavender

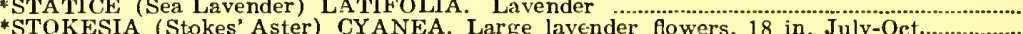

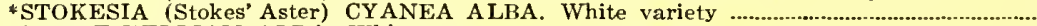

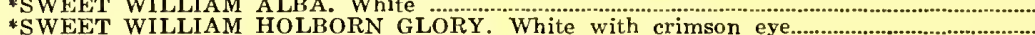

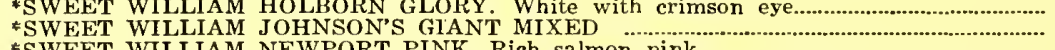

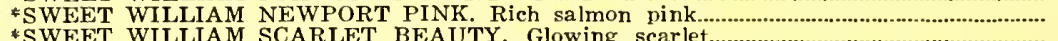
*SWEET WILLIAM SCARLET BEAUTY. Glowing scarlet.............................................. *SWEET WILLIAM LATIFOLIUS ATROCOCCINEUS FL. PL. Semi-double, deep crim*TRITOMA (Red Hot Poker) HYBRIDUM. Red and orange shades *TRITOMA (Red Hot Poker) HYBRIDUM PERPETUAL FLOWERING Red and orang

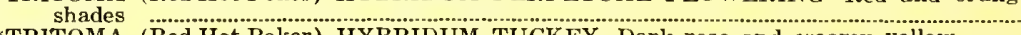
*TRITOMA (Red Hot Poker) HYBRIDUM TUCKEY. Dark rose and creamy yellow............ *TRITOMA (Red Hot Poker) PFITZERI. Orange scarlet...................................................... *VALERIANA (Garden Heliotrope) COCCINEA. Soft pink to purplish red. 3-4 $\mathrm{ft}$. June

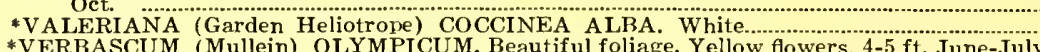
*VERBASCUM (Mullein) OLYMPICUM. Beautiful foliage. Yellow flowers. 4-5 ft. June-July *VERBASCUM (Mullein) PHOENICEUM. Beautiful. All colors, from deep yellow and

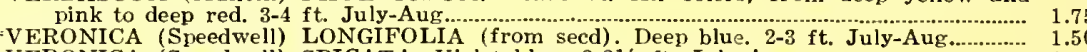
YUCCA (Adam's Needle) FILAMENTOSA. Large spikes of white flowers, 4-6 ft. June.

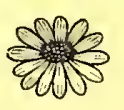

\section{A FEW POINTERS TO REMEMBER}

All our Perennials are strong, field-grown stocks.

All our Perennials have been transplanted into the field.

All our Perennials are shipped as dug from the field.

All our Perennials are shipped from young stocks; no old, overgrown stock.

All our orders are checked three times before going in the box. All we work for is a larger Perennial business all the time. 



\section{HARDY PHLOX \\ (Our National Garden Flower)}

We possibly grow the best Phlox that is being handled, because of our splendid soil and our liberal selection. Our collection of varieties is without question the best that is being grown anywhere. We believe in Phlox. They are the best garden flowers we have. Our Mr. P. Weller went to Europe last year and picked up a lot of new and splendid varieties, some of which we are listing this year, and many more to come in the future. All our Phlox are strong, one-year-old field-grown plants with all the roots
as dug. No better plants on earth.

AFRICA. Purplish carmine, blood red eye. 30 inch

ANNA COOK. Soft pink, very beautiful. 30 inch

ASIA. Pale lilac with red eye. 24 in

nge-red, 24 inch

B. COMTE. Brilliant purple. 24 inch......... Phlox. 36 inch

BRADESMAD. White, crimson eye, 40 inch

BRILIANT (New) Large trusses of fiery scarlet. $30 \mathrm{inch}$

CHAMPS ELYSEE. Dark purnle. 24 inch

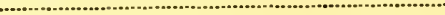

COQULLICOT. Orange-red. Very striking.

.

ENCHANTRESS. Bright salmon pink, with red eye..........

EUROPE. Pure white with carmine red

FER BU CORT. Pure white. 32 inch...........

FEUERBRANDT. (Firebrand) Clear orange-scarlet. Extra. 36 inch

GENERAL VON HEUTZ, Salmon-red, white and carmine eye 36 inch

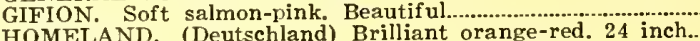

IMPERATOR. (New) Brilliant dark carmine with darker eye. 32 inch

*INDEPENDENCE. Pure white. 30 inch..

*ISABEY. Salmon-pink. 36 inch....................

LA VAGUE. Warm mauve with carmine eye

*LORD RALEIGH. Dark lilac. 28 inch.

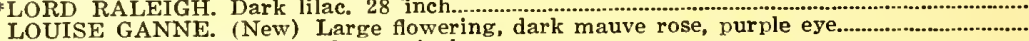

MILLY VON HOBOKEN. A fine, clear, pink without an eye, large fower. 36 inch..

MISS LINGARD. Early white, 36 inch..............

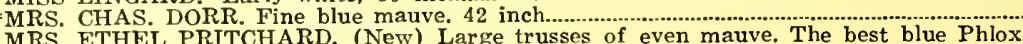

MRS. ETHEL PRTCHARD. (New) Large trusses of even mauve. The best blue Phlox.

MRS. JENKINS. Pure white. 30 inch

rosy-red. 36 inch

MRS. RUTGERS. (New) Light lilac pink, darker center.............................................................

MRS. SCHOLTEN. (New) Dark salmon pink. Large flower

OBERGARTNER WITTIG. Lavender rose. A fine Phlox

*PANTHEON. Carmine-pink. 30 inch.

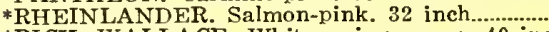

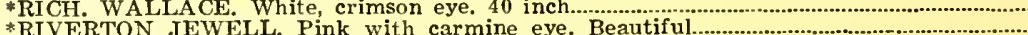

RIVRTON JEWELL. Pink with carmine eye. Beautiful

*RYNSTROM. Deep pink. 48 inch................................

SPECIAL FRENCH. Fine blush pink with salmon eye. Largest.

*THOR. Deep salmon. 40 inch

VON LASSBURG. Best white. 28 inch

W. C. EAGON. Beautiful soft pink, extra fine

W. KESSELRING. (New) Large violet, purple eye. 24 inch......................................................

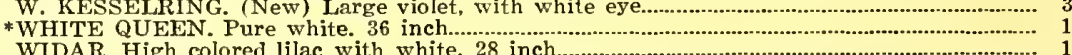

For large quantities, ask for special quotations. Varieties marked with (*) $\$ 70.00$ per 1000

Per 10 Per 100

\section{PEONY PRICES FOR FALL, 1929}

Three to five eyes, plus Roots. From two-year-old plants only. The best money can buy. Those marked with $\mathrm{a}^{*}$ suited for Decoration Day cutting.

Per 10 Per 100

ALBATRE. (Avalanche) (Crousse. 1895) Late white, flecked crimson. Very fragrant, ALBERT CROUSSE. (Crousse. 1893) Late, rosy white, fragrant, free bloomer. Perfect in form. Extra fine............................................................................................

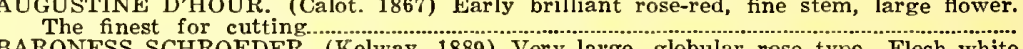
bud, bleaching to white when open. The finest white peony bud, bleaching to white when open. The finest white peony .............................................
BOULE DE NIEGE. (Calot. 1867) Erect strong grower, milk white with sulphur center. COURONNE D'OR. (Calot. 1873) This is the famous crown of gold. Snow white reflect-

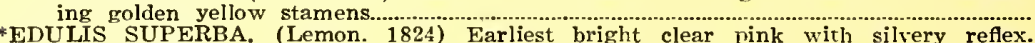

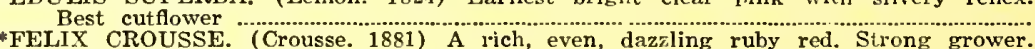
*FELIX CROUSSE. (Crousse. 1881) A rich, even, dazzling ruby red. Strong grower. Finest variety for cut flowers
*FESTIVA MAXIMA. (Mielez. 1851) Very early, pure white, flecked crimson. Best pop-

Kular white brilliant without a trace of purple. A flower of rare beauty. Immense in size, globu-

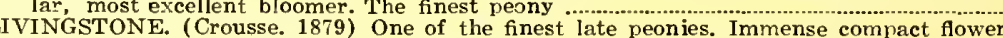

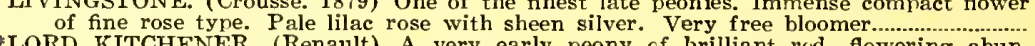

*LORD KITCHENER. (Renault) A very early peony cf brilliant red, flowering abundantly. Earliest red peony and a promising variety...................................................
*MAD. CALOT. (Meilez. 1856) A wonderful free white flowering peony. Overlaid with

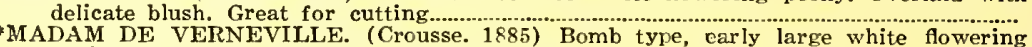
variety with four red flakes on center petals........................
*MADAM DUCELL. (Mechin. 1880) Bomb type, both guards and bomb of a solid silvery pink color. Holds its form and color to the end. For cutting as good as Mons. Jules

MADAM EMILE LEMOINE. (Lemoine. 1899) Extra large imbricated round fowers. On first opening glossy white, overlaid with a sheen of tender satiny pink, covered with MAD. LEONIE CALOT. (Calot) Rose type. Late midseason. Belicate rose white with soft

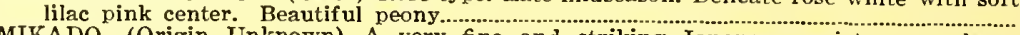
MIKADO. (Origin Unknown) A very fine and striking Japanese variety, very large blooms. Guards of very broad petals of brilliant red. Center petals long and narrow of bright golden yellow, making it a striking flower.......................................................
MONS. JULES ELIE. (Crousse. 1888) Early, glossy lilac pink color, shading to deeper

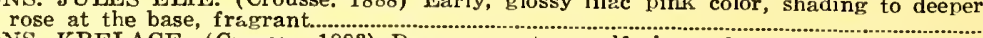
MONS. KRELAGE. (Crousse. 1883) Deep currant or solferine red, amaranthine center. Large flat fowers, perfect in outline
PRESIDENT TAFT. (Syn. Reine Hortense) (Blaauw. 1909) Early, midseason, fragrant, should be cut in the bud to obtain full attractiveness. Semi-rose Hydrangea pink, QUEEN VICTORIA. (Eugene Hort. 1830) Bomb type, early midseason. A wonderful

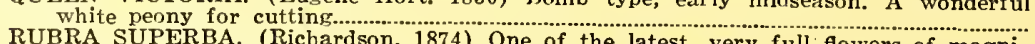
RUBRA SUPLRBA. (Richardson. 1874) One of the latest, very full flowers of magniSARAH BERNHARDT. (Lemoine. 1906) 'The strongest growing of all without exception.

Very large and perfect in form. Color apple-blossom pink, silvery tipped...................
TRIOMPHE DE L'EXPOSITION DE LILLE. (Calot 1865) Midseason. fowers of a fresh Hydrangea pink color, splashed with darker tints of violet rose. Beautiful

RED UNNAMED 


\section{GLADIOLI For Forcing and Outdoor Planting}

\section{OUR SUPERB STOCK}

As pioneers in Gladiolus growing, and having been among the first to introduce "Gladioli for forcing," much experience in nroducing "A Sunerior Kind of Bulb" that the ne dwelled unon. Our hundreds of satisfied custmmers are our best testimonials. Fvery year we have been able to imnrove our stnck through

Those suitable for forcing are marked with a $(*)$.
Thent

*AMERICA. Soft, flesh nink, tinted lavender

ANNA EBERIUS. Dark, velvety purple

CATHARINE. Wonderful grayish blue.

CHICAGO WHITE. Pure white with lavender markings

CRIMSON GLOW. Brilliant crimson, extra large

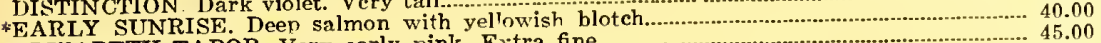

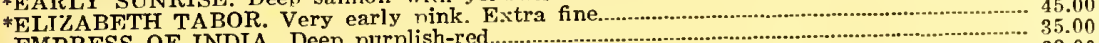

EMPRESS OF INSIA. Deep purk with scarlet b'otches........................................................ 32.00

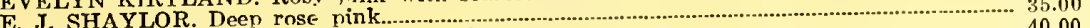

*FLORA. Beautiful golden yellow. Very large

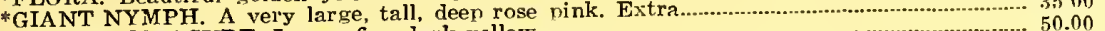

GOLDEN MEASURE. Large, fine dark yellow

*HALLEY. Delicate salmon pink with cleamy hlotch

HERADA. Pure mauve witl deeper mirkings

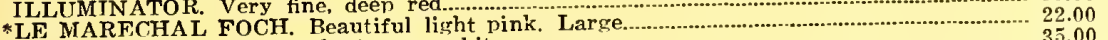

*LE MARFCHAL FOCH. Beatiful light pink. Lar............................................................. 35.00

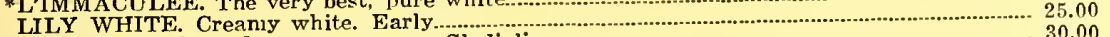

*LOUISE. The lavender queen among Gladioli ......................................................................... 30.00

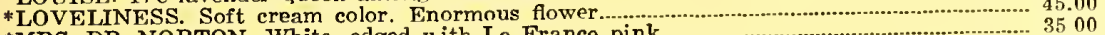

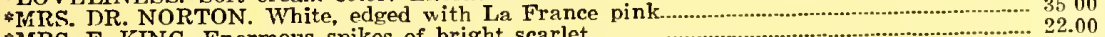

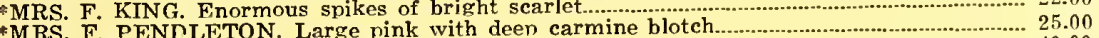

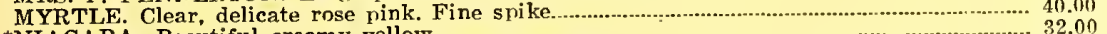

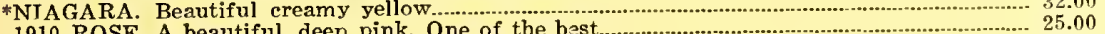

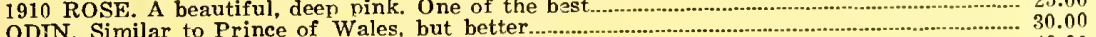

ODIN. Similar to Prince of Wales, but better

ORANGE GLORY. Deep orange with carmine blotch

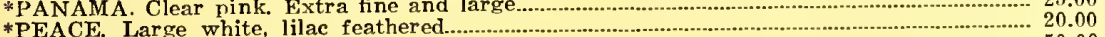

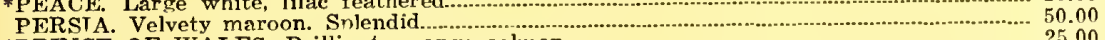

PRINCE OF WALFS. Brilliant, orange salmon

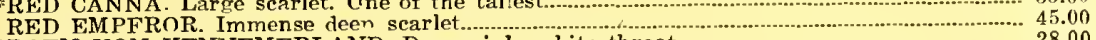

*ROEM VON KFNNEMERLAND. Deep nink. white throat

ROSE ASH. Ashes of roses. Beautiful buff color. Tall.................................................... 40.00

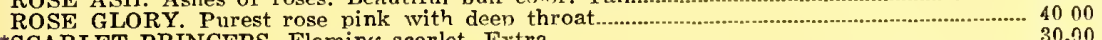

*SCARLET PRINCEPS. Flamine scarlet. Extra

*SCH WABEN. Canary yellow shading to sulphur................................................................ 28.00

*TYCHO ZANG. Very large salmon pink, Extra fine

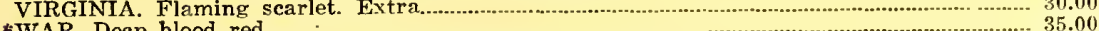

*WAR. Deen blood red.... Halley. Berutiful shade of light pink

YELI,OW HAMMFR. Pure yellow. Strong grower ............................................................... 35.00

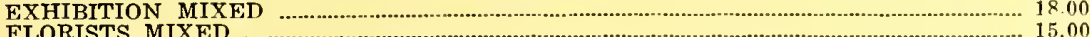

FLORISTS MIXED

PRIMULINOUS VARIETIES

*ALICE TIPLADY. Beautiful shade of orange

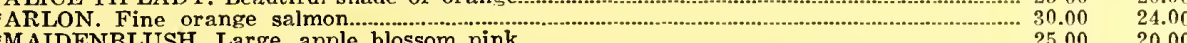
*MAIDENBLUSH. Large, apple blossom pink............................................................................. 25.00 20.00

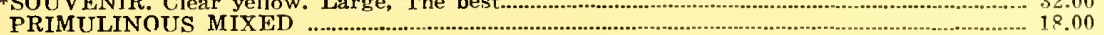

DUTCH AND AMERICAN GROWN BULBS FOR FALL, 1929

We import our bulbs from one of the best growers in Holland, and have given satisfaction wherever we have sold them. Dutch bulbs are either shipped from Chicago or Holland, Mich. Prices quoted are f.o.b. either station.

Smallest lots 100 of one kind.

\section{HYACINTHS}

BEDDINC SIZE, 14-15

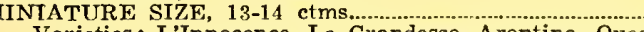

Varieties: L'Innocence, La Grandesse, Arentina, Queen of the White, Gertrude, Lady

\section{SINGLE FARLY TULIPS}

CRAMOISI BRILLIANT. Deep red.

DIANA. Pure white. Late.

LA REINE. Blush white.

PRINCE OF AUSTRIA. Orange-red

ROSE GRIS-DE-LIN. Light pink.

WHITE HAWK. Pure white, Early.

YEILLOW PRINCE. Pure yellow

\section{SINGLE LATE OR COTTAGE TULIPS}

BOUTON D'OR. Golden yellow...................

FAIRY QUEEN. Heliotrope with yellow....

GESNERIANA SPATHULATA. Scarlet-red..

INGLESCOMBE YELLOW. Golden

INGLESCOMBE PINK. Fine pink.....................

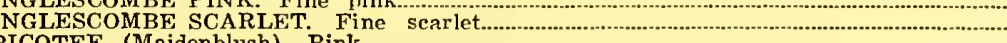

PICOTEE (Maidenblush).

CHOICF M

\section{DARWIN TULIPS}

BARONNE DE LA TONNAYE. Bright rose..

BARTJGON. Brilliant red. Early

CLARA BUTT. Salm

FARNCOMBE SANDFRS. Bright scarlet

GRETCHEN. Soft pink

PAINTED LADY. Cream-white......

PRIDE OF HAARLEM. American Beauty color

REV. EWBANK. Light Hcliotrope

WHITE QUEN. Brown-black.

ZULU. Violet black........................
CHOJCE MIXTURE. All colors

DOUBLE EARLY TULIPS

$3.80 \quad 33.00 \quad$ COURONNF D'OR Orange-yellow $\begin{array}{lll}3.00 & 26.00 \\ \text { BOULE DE NEIGF Pure white. } 3.80\end{array}$

MISCELLANEOUS BULBS

CROCUS. Blue, white, striped and yellow.

GRAPE HYACINTH

SNOLDROPS

PAPER WHITE. 13-14 ctms

LILIUM CANDIDIN Per 10 Per 100

$\begin{array}{lcccr}\text { LILIUM CANDIDUM } & \ldots . . . . . . . . . . . . . & 2.50 & 20.00 \\ \text { LILIUM } & \text { REGALE. 6-7 } & \text { inches........ } & 4.00 & 30.00\end{array}$

LILIUM REGALE. 4-5 inches...

25.00

25.00

25.00
30.00

25.00

34.00

35.00

2300

23.00

26.00
23.00

23.00

23.00

23.00

22.00

25.00

25.00

2500
27.00

25.00

25.00

25.00

25.00

25.00
28.00

30.00

24.00

3800

33.00

25.00

25.00

25.00

50.00

20.00 


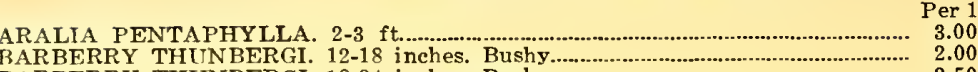

BARBERRY THUNBERGI. 18-24 inches. Bushy.

CORNUS STOLONIFFRA. $2-3 \mathrm{ft}$.

DEUTZIA CRENATA. $2-3$ ft.

DEUTZA CRENATA. 2-3 1 . .............

DEUTZIA PRIDE OF ROCHESTER, $2-3 \mathrm{ft}$

DEUTZIA PRIDE OF ROCHESTFR $3-4 \mathrm{ft}$

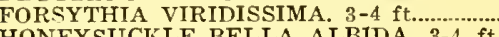

HONEYSUCKLE BELLA ALBIDA. 3-4 ft.........

HONFYSUCKLE MORROWI 2-3 ft

HONEYSUCKLE TARTARIAN PINK. $2-3 \mathrm{ft}$

HONFYSUCKLE TARTARIAN PINK. 3-4 ft...

HONFYSUCKLE TARTARIAN IVHITE. $2-3$ ft.

HONEYSUCKLE TARTARIAN WHITE. 3-4 $\mathrm{ft}$

KFRRIA JAPONICA. 12-18 inclies

KFRRIA JAPONICA. $24-30$ inches.

KFRRIA JAPONICA. $24-30$ inches............................

PHILADELPHUS CORONARIUS. $2-3 \mathrm{ft}$

SNOWBFRRY WHITE. $2-3 \mathrm{ft}$.

SPIRAFA BILLARDI ROSEA $2-3 \mathrm{ft}$

SPIRAEA BILLARDI ROSEA. 3-4 ft.

SPIRAEA DOUGLASI. $3-4 \mathrm{ft}$.

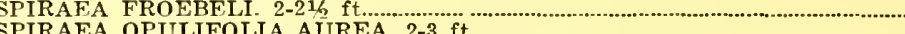

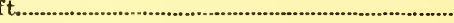

SPIRAF,A VAN HOUTTE. 3-4 f

VIBITRNUM OPULUS. $2-3 \mathrm{ft}$

WFTGFLIA AMABILIS. 2-3 ft.....

A

WEIGELIA ROSEA. 2-3 ft

2-3 fí..

\section{CLIMBING VINES}

AMPFLOPSIS QUINOUEFOLIA, 2 year. No. 1

AMPELOPSIS VFITCHI. 2 year No, 1

CLEMATIS TANGUTICA (New). Flowers solitary, bright yellow, nodding, 2

inches across, growing ten feet high. June-July. Later in the summer large

heads of feathery fruits. Very handsome.

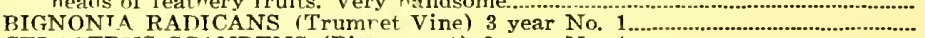

CFLASTRIS SCANDFNS (Bittersweet) 2 year No. 1...................................................... 3.0

HONEYST TKKLE HALLEANA 2 vear No. 1 1.......................................................... 2.50

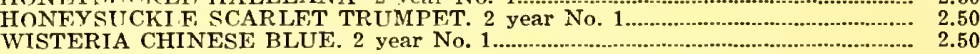

\section{ORNAMENTAL TREES}

SOFT OR SILVFR MAPLE. $8-10 \mathrm{ft}$

\section{EVERGREENS}

IUNIPFRUS PFITZERIANA. Spreading. 2-21/ $\mathrm{ft}$ across.

JINTPERUS PFITZFRTANA. Snreading. $21 / 2$ ft. across.

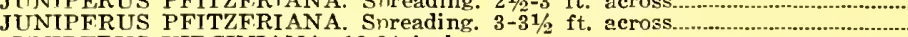

JIINIPFRUS VIRGINIANA. 18-24 inehes.

JTNIP RUS VIRGINIANA $21 / 2$ ft

(1)

JUNIPFRUS VIRGINIANA. $3-4 \mathrm{ft}$.

JUNIPFRUS VIRGINIANA. 4-5 $\mathrm{ft}$.

BOXWOOD. For pots, small tubs or window boxes.

BOXWOOD. Fine, Bushy plants. $8-10$ inches

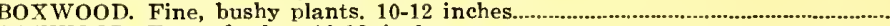

BOXWOOD. Fxtra bushy. 10-12 inches

Extra bushy. 12-14 inches

(a) boxwood, 8-10 inches.

\section{ROSES}

F. J. GROOTENDORST. Rugosa Baby Rambler. Crimson......................

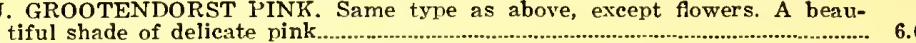

Per 10 Per 100 Per 1000 $8.50 \quad 75.00$

\subsection{0}

300.00

350.00

75.00
125.00

175.00

30.00

60.00

35.00

50.00

\section{WELLER'S FORCING STOCK}

\section{Grown Well Cured Properly Graded Generously}

One of our specialties is serving the florists, and it is therefor not surprising that we have studied their requirements. Our roses are exceptionally fine, strong, heavy flowering canes, well selected and low budded. Our spiraea have always been satisfactory and we have grown them for a number of years.

\section{ROSES FOR FORCING-(FEAVY XXX) BABY RAMBLER VARIETIES}

ENTTH CAVELL. Dark maroon.

EILLFN POULSEN. Large, clear pink.

ERNA TESCHENDORFF. Deep crimson

GOLDEN SALMON. Beautiful shade of salmo

IDFAL. Deen maroon.

I.F.VEVASSEUR. Crimson

ORANGE SALMON. Beautiful orange.

TRIOMPH DE ORLFAN ?

\section{CLIMBING ROSES}

CRIMSON RAMBLER. Crimson

DOROTHY PERKINS. Pink...........

EXCELSA. Red $1 . \ldots \ldots$. Cherry red

MARIE GOUCHAULT. Identical with Dorothy Perkins, excepting color, which is a clea

geranium-nink without magenta shadings. Very free and early....... PAIILS SCARLET. Scarlet.

\section{HYBRID PERPETUALS}

FRAU KARL DRUSCHKI. White....

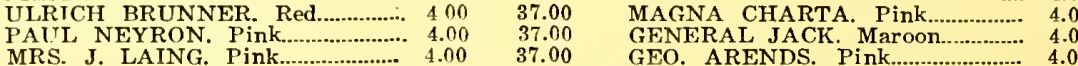
SPIRAEA (ASTILBE) FOR FORCING - (HEAVY CLUMPS) AMERICA. Beautiful violet blue. GTA

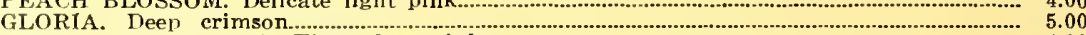
QUEEN ALEX CNDRA. Fine, clear pink $\ldots$ DIELYTRA SPECTABILIS. (Bleeding Hearts) .................................................................................... 4.50 DELPHINIUM (LARKSPUR) FOR FORCING

DELPHINIUM BELLADONNA. Sky blue. Strong 2 year...................................................... 10.00 DELPHINIUM BELLADONNA. Sky blue. Strong 1 year........................................................ 8.00 DELPHINIUM BELLAMOSA. Deep blue. Strong 1 year.................................................................. DELPHINUM ENGLISH HYBRIDS. 2 year...................................................................................... 12.00 DELPHINIUM ENGLISH HYBRIDS. 2 year. 


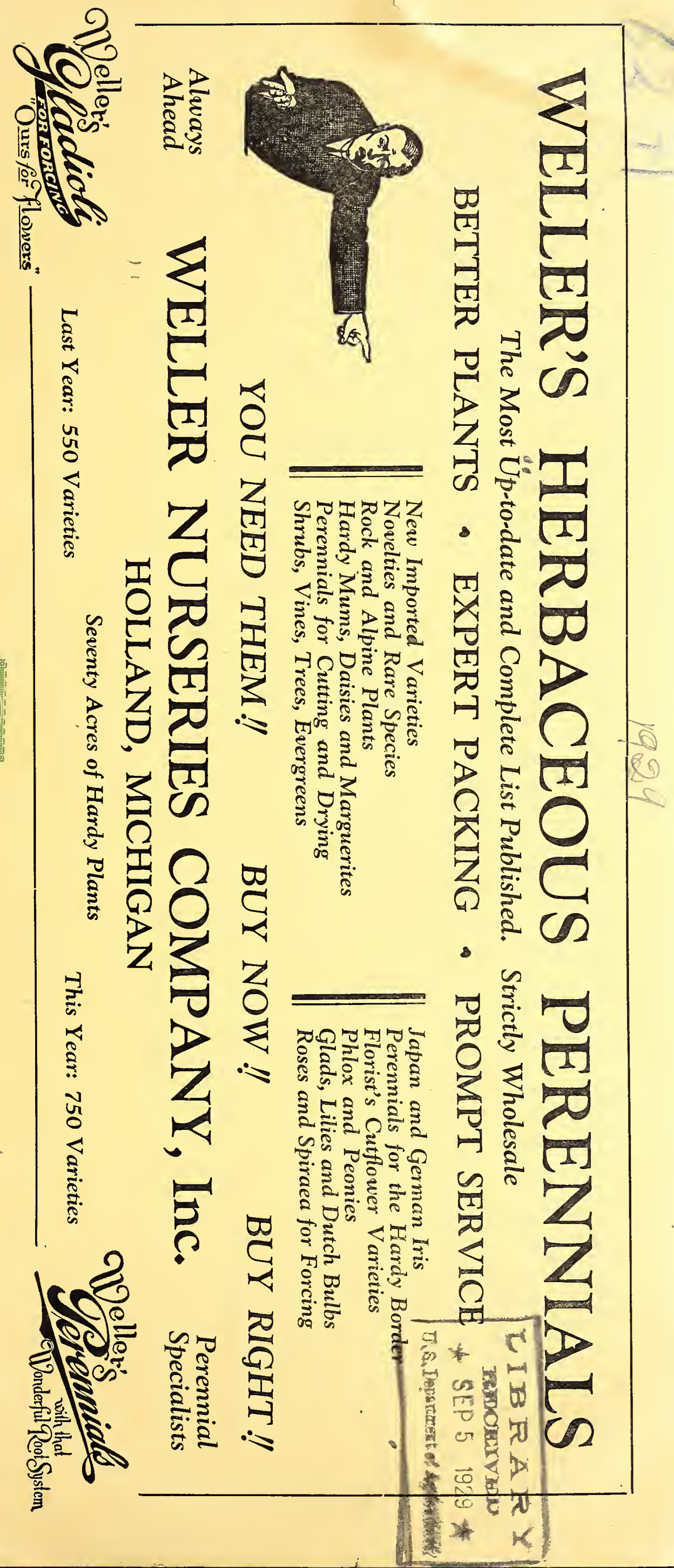


